\title{
Integrated diabetes care: The Association of British Clinical Diabetologists (ABCD) national survey report
}

\author{
DINESH NAGI, SUSANNAH ROWLES, ANDREW MACKLIN, UMESH DASHORA, HEATHER OLIVER, \\ DIPESH PATEL, ON BEHALF OF THE ABCD
}

\section{Executive Summary}

A national survey on integrated diabetes services was carried out by the Association of British Clinical Diabetologists $(A B C D)$ during the COVID-19 pandemic and has provided some very useful insights into the current state of integration to deliver a joined-up diabetes service in the UK.

This survey was carried out during the second half of 2020 and explored three main areas: (1) current state of clinical integration between primary and secondary (specialist) diabetes services; (2) the state of IT integration among the diabetes IT systems and hospital-based electronic patient records (EPR) and between hospital and primary care; (3) to ascertain the membership of their views on a 'one-stop service' for collecting annual review data for diabetes and the potential barriers to achieve this. The results presented are a summary of the survey, while the full unedited survey report, especially on the qualitative aspects, is available to $A B C D$ members.

The survey was mailed to 518 individuals, of which 431 (83.2\%) were consultants and $53(10.2 \%)$ were specialist registrars. Of the 83 replies received, $98 \%$ were from consultants and the responses represented a total of 73 hospital diabetes services.

The findings of this survey revealed that full integration of clinical services among primary care and specialist diabetes teams is uncommon, although there are good examples of clinical integration in different formats. In a number of areas, primary care and specialist diabetes services continue to work in silos despite a universal recognition that integrated services are desirable and are likely to improve quality of care. Clinical leadership, resources and buy-in from those who commission services were deemed important factors to help improve the development of integrated care systems.

In hospitals with dedicated diabetes IT systems the infor-

\section{$A B C D$ executive}

Address for correspondence: Dr Dinesh Nagi

Edna Coates Diabetes and Endocrine Unit, Pinderfields Hospital,

Aberford Road, Wakefield WF1 4DG, UK

Tel: +44 (01924) 213594

E-mail: d.nagi@nhs.net

https://doi.org/10.15277/bjd.2021.325 mation flow from these diabetes systems to the EPR was not universal, raising concerns that vital information about an individual's diabetes may not be available to other hospital clinical specialities at the time of delivery of care, posing a significant clinical risk. IT integration among primary and specialist diabetes teams in England was only available in certain areas and was mostly based around the use of SystmOne.

The survey also identified a diversity of opinions regarding the current arrangements of the Quality Outcome Framework (QOF), where GPs are incentivised to collect data for annual review of routine diabetes care. Many were of the opinion that annual review processes should be performed by clinical teams who are tasked to deliver diabetes care to the individual, while others felt that the status quo should continue with primary care GPs being responsible. A one-stop service for eye screening for diabetes and other annual measurements nearer to people's homes was identified as an improvement, but several logistic barriers were identified.

We recognise the limitations of any survey which expresses opinions of participants. However, we believe the present survey represents a significant proportion of diabetes units in the UK and provides insights into the current state of integrated services in diabetes. There are significant learnings for diabetes communities, and the information can be used to improve and galvanise delivery of integrated diabetes care in the UK.

Br J Diabetes 2021;21:272-280

Key words: diabetes, integrated services, survey, annual review process, primary care, specialist diabetes services

\section{Introduction}

\section{Context of COVID}

The Association of British Clinical Diabetologists ( $A B C D$ ) has been active in producing several guidelines and reports during the COVID-19 pandemic. These reports were mostly designed to provide guidance for specialist diabetes teams to enable the delivery of diabetes care at the peak of the viral pandemic, where the emphasis was to support acute services for people admitted to hospital, ${ }^{1,2}$ to support people with diabetes at high risk of poor outcomes $^{3}$ and to alter systems to focus care on those deemed at high risk. 
One positive result of the pandemic was that it has afforded clinicians in the UK and worldwide the opportunity to innovate to help support patients in new ways. It is clear to healthcare professionals and the wider NHS that provision of clinical services will never be the same again, and opens the possibility of shaping a 'new normal' for clinical service provision. ABCD has also published its own guidelines on individual risk stratification and recovery of diabetes services. ${ }^{2}$

With the delivery of a widespread vaccination programme and proposed 'roadmap out of lockdown', ABCD was keen to produce a 'real-time' piece of work to help promote positive evolution of diabetes services post COVID, to ensure the learning and innovation during the pandemic becomes embedded and is not lost. ${ }^{4,5} \mathrm{ABCD}$ would regard a return to 'business as usual' arrangements to deliver diabetes services as a wasted opportunity for long-term change to reconfigure services.

This survey was conceived, designed and piloted by the $A B C D$ Executive team and then sent to the organisation's membership. The membership comprises four nations (England, Scotland, Northern Ireland and Wales) and represents a diversity of practice including small district hospital teams, community services and larger academic institutions.

The aim was to benchmark diabetes services as they are now, drawing on the expertise and experience of those who have shaped them to date, and asking them what a better future would look like and how it could be achieved.

The survey had three sections focusing on evidence-based key elements of successful delivery of diabetes services:

(1) integration of clinical services among specialist and primary care teams (Section A)

(2) the current state of IT support available to the specialist diabetes teams to support clinical integration (Section B)

(3) how the current provision of an annual review process for the collection of vital data may be improved (Section C)

\section{Definition of integration}

The concept of an 'integrated diabetes service' is not new and means different things to different healthcare professionals, managers and health policy makers. Integrated diabetes care involves both integration of a healthcare system and coordination of services around a patient. "An approach that seeks to improve the quality of care for individual patients, service users and carers by ensuring that services are well co-ordinated around their needs"..$^{6-8}$ In essence, diabetes integration is the whole health community joining in partnership to 'own' healthcare delivery and outcomes of patients with diabetes in each locality. ${ }^{8,9}$

However, for the context of this survey, we defined integrated diabetes care as "clinical care in a given health economy where the delivery of diabetes care is seamless among specialist and primary care and is well supported with IT systems, where planning, delivery and learning from these services is joined up with sharing of information, and where services are efficient and provide value for money". Delivering integrated care is challenging for numerous reasons, including the complexity of diabetes care and organisations working in silos focusing on their own priorities which are not necessarily aligned with each other. ${ }^{10,11}$ Moreover, measuring the success of an integrated care system is extremely challenging; however, several key indicators to measure the success of clinical integration have been proposed. ${ }^{11}$

The aims of this survey were to gather information and intelligence at a national level for each of the above three elements and to produce a summary to inform future recommendations and catalyse discussions around the topic. A definition and explanation of integrated care was provided to help complete the survey questions related to this (Section A).

\section{Survey methods}

The first draft of the survey questions was written by one of the authors (DN) with contributions and further refinements by the $A B C D$ executive team, and was shared with Diabetes UK and NHS England before it was disseminated via email to diabetologists who are members of $A B C D$. Due to the nature of this survey, we had invited open comments from participants to gather as much qualitative information as possible which is included in this report. The responses to the survey were handled by the $A B C D$ secretariat and preliminary data analyses were produced.

\section{Results}

The survey was sent to 518 individuals comprising 431 (83.2\%) consultant grade, $53(10.2 \%)$ specialist registrars, 30 others and two retired healthcare professionals, one paediatric diabetologist and one dietician. Of the 83 replies received, $98 \%$ were from consultants. Although the original survey response was $17 \%$, we believe that it represents 73 hospital-based diabetes services. The number of those who responded by region is shown in Table 1.

\section{Quantitative results}

The results are given as absolute numbers and percentages which are rounded up to the nearest number. Where there was more than one potential answer, percentages exceed $100 \%$ (see survey results Sections A, B and C).

Table 1 Responder number by region

\begin{tabular}{cl}
\hline Region & Number \\
East Midlands & 2 \\
East of England & 5 \\
Greater London & 14 \\
North East & 1 \\
North West & 7 \\
South Central & 5 \\
South East & 7 \\
South West & 11 \\
West Midlands & 4 \\
Yorkshire \& Humber & 6 \\
Northern Ireland & 1 \\
Scotland & 4 \\
Wales & 5
\end{tabular}




\section{Survey results}

Section A: Integrated Care (Figure 1a-c)

Do you think that your secondary care-based service is integrated with primary care?

Yes

No

Don't know

What is the nature of this clinical integration?

Fully integrated service

Partial integrated service

Little clinical integration

Do you think that the wider diabetes services (primary and secondary care) work in a joined-up way?

Yes

No

Don't know

Do you have a regular review and evidence (including collection of evidence)?

Yes

No

Of those who responded Yes to the above question, we asked if this has made a difference Yes

Don't know

Are you planning to have a clinically integrated service with primary care within the next 12 months?

Yes

Already in place

Has the COVID-19 pandemic (only those who responded Yes)

Speeded up planning

$4(18 \%)$

$16(72 \%)$

$2(10 \%)$

Made no difference to planning

Section B: Diabetes IT (Information Technology) Systems (Figure 2a-d)

Do you have an EPR (electronic patient record) in your hospital?

Yes

$59(72 \%)$

No

$23(28 \%)$

Do you have a dedicated diabetes IT system within your Trust?

Yes

No

Was it commercially purchased or was it built in-house?

Commercially purchased

Built in-house

$28(72 \%)$

$11(28 \%)$

Who can access this system?

All clinical users (read only)

All clinical users (read/write)

Those who have been authorised to log in

Only members of the diabetes MDT

How does your diabetes IT system interact with EPR?

Both systems work as standalone systems

Diabetes system data visible from the EPR

Diabetes data accessible from the EPR as a read-only view which

is not displayed by default

Bidirectional connected to share information with the EPR

Do you have full, partial or no IT integration of diabetes IT systems with primary care IT systems?

Read only

Read/write

One directional or bidirectional

$11(29 \%)$

$6(16 \%)$

Is messaging and tasking supported?

$20(51 \%)$

$7(18 \%)$

$25(68 \%)$

$4(11 \%)$

$5(14 \%)$

$3(7 \%)$
Does the community access apply to all community settings or

only selected ones?

In your view, how important is IT integration with primary care to help deliver seamless care?

Crucial

$29(74 \%)$

Important

$10(26 \%)$

Does your locality have plans for IT integration with primary care in the near future (ie, <12 months)?

Yes

$37(47 \%)$

Don't know -

If yes - has this been prompted by the COVID-19 pandemic?

Yes $1(4 \%)$

No $21(82 \%)$

Don't know $1(4 \%)$

What are the main barriers to having an integrated IT system across specialist and primary care? (Figure 3)

Lack of prioritisation by senior decision makers 49 (39\%)

Funding issues $\quad 35(28 \%)$

Lack of suitable systems (ie, have tried and failed) $\quad 18(15 \%)$

Other

$20(16 \%)$

Section C: Annual Diabetes Reviews

The existing arrangements are adequate and should continue without any change

Yes

No

$17(21 \%)$

Don't know $\quad 5(6 \%)$

When looking at the process of annual review, what are the potential alternatives?

Annual reviews should be performed by the clinical teams primarily responsible for regular follow-up for diabetes. For example, people with type 1 or type 2 diabetes under hospital follow-up should have

Primary care should be responsible for review of all patients Annual review arrangements should change and should take place as a 'one-stop shop'

Looking at the logistics of a 'one-stop shop'

to collect annual review data, which would be your preferred option?

At the time of retinal screening but delivered

by a dedicated and trained team

At a different time from the retinal screening

but in the community setting

If a 'one-stop shop' service could be delivered in the community set-up (nearer to home), where could these be located?

GP surgeries

$39(34 \%)$

Pharmacies

$15(13 \%)$

Opticians

Supermarkets (eg, ASDA)

Any of the above

$9(8 \%)$

Other location (please specify)

$8(7 \%)$

Do your patients have direct access to any of their healthcare information (or healthcare records)?

$33(41 \%)$

No

$37(46 \%)$

Don't know 
Figure 1. Results of the integrated diabetes services

a. Do you think that your secondary care based service is integrated with primary care?

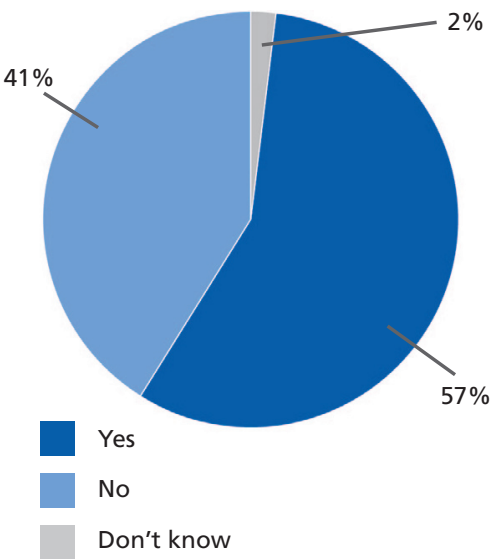

b. What is the nature of this clinical integration?

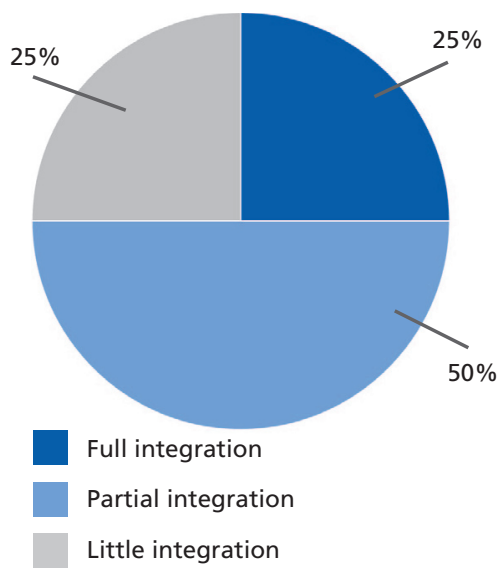

c. Do you think that the wider diabetes services (primary and secondary care) work in a joined-up way?

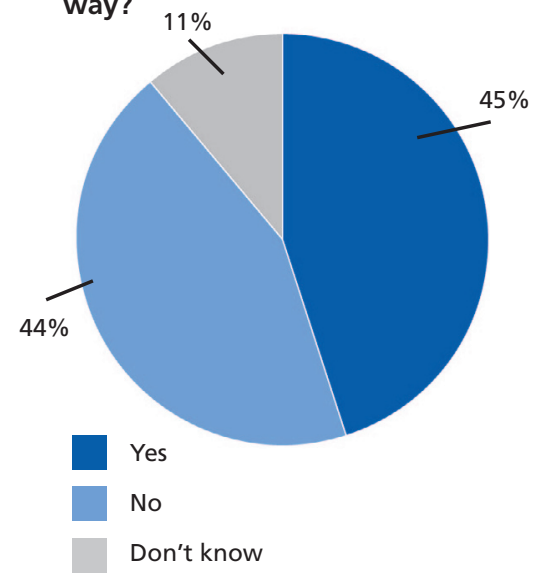

Figure 2. Results of the IT integration within and outside the organisation a. Diabetes IT (Information Technology) Systems: Do you have an EPR (Electronic Patient Record) in your hospital?

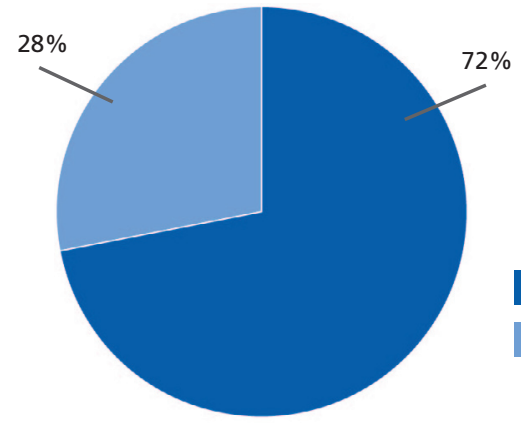

c. Was it commercially purchased or was it build "in house"?

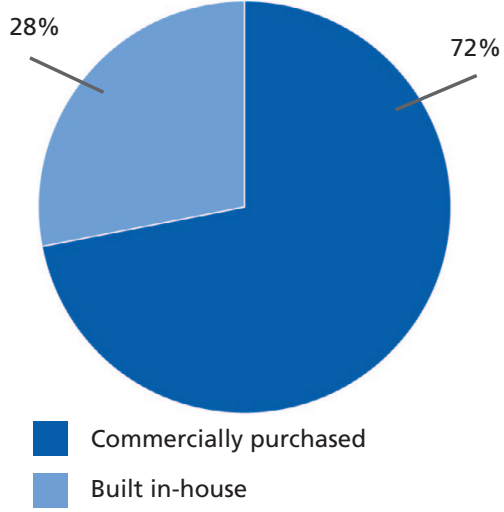

b. Do you have dedicated Diabetes IT system in your trust

Yes No

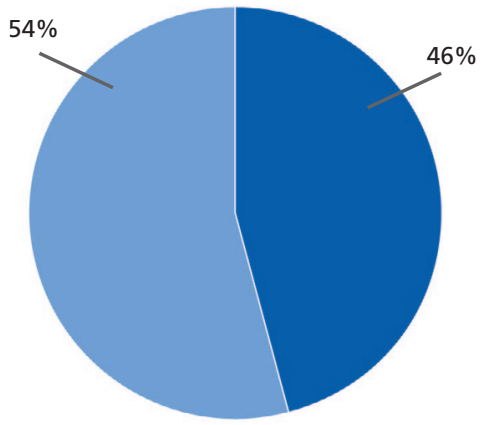

d. Does your locality have plans for IT integration with primary care in the near future? i.e. <12 months?

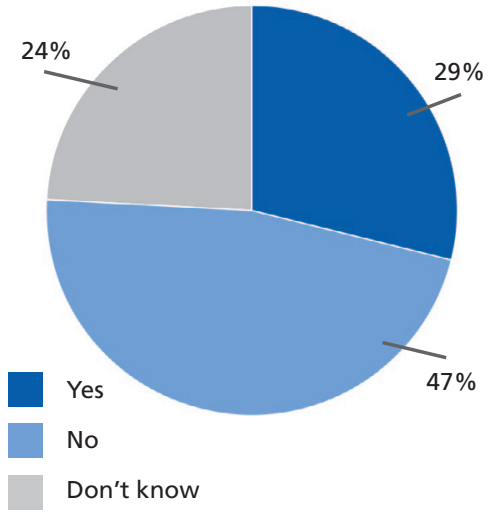

Figure 3. What are the main barriers to having an integrated IT system across specialist and primary care?

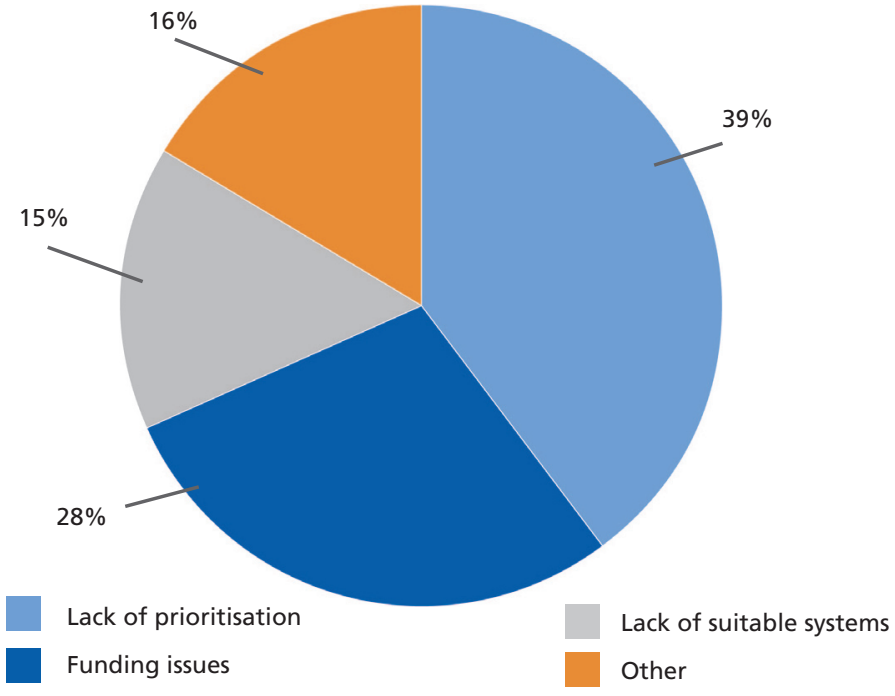




\section{Box 1 Levels of integration seen in the survey}

Different levels of integration among specialist and primary care services exist within the UK

1. Intermediate Diabetes Services Model

- There are examples of community diabetes teams (as intermediate services) which provide a link between primary care and specialist diabetes services.

- Intermediate services are predominantly made up of community-based Diabetes Specialist Nurses (DSNs), but some services include communitybased diabetologists.

- In some services consultants are integrated with community diabetes teams but the diabetes nurse teams are not.

- Where intermediate services are run by DSNs, support is provided from the specialist teams based in hospital, with extremely close liaison between the workforce to some services where DSN working was almost in total isolation.

- In some services DSNs were based with the specialist teams but were responsible for supporting primary care for less complex cases including insulin start and injectable therapies.

2. Primary care support by hospital-based specialist services

- Diabetes services are planned together with clear commissioning of the level of services provided by the specialist teams (ie, Super Six Model, Derby Model, Wakefield integrated Services), where specialist diabetes teams (consultants and DSNs) provide regular joint sessions in primary care including virtual reviews.

- Primary and specialist care hold regular MDT discussions of complex cases including telephone or e-consultation.

- Specialist and primary care teams meet regularly outside the clinical context to learn together and ensure regular clinical updates regarding new therapies.

3. Integration in Scotland

- Clinical data are shared between primary and secondary teams. There is good dialogue between primary and secondary care clinicians for some patients, but much better dialogue and alignment of practice would be better. Also, centralised complication screening for all patients is our desire but needs funding at the Health Board level since primary and secondary care presently all do their own screening (or not, since COVID). Scotland has a very different model of care from England, hence many more patients are seen in secondary care clinics.

\section{Qualitative results}

The survey generated significant information on the views, ideas and experiences of diabetes specialists, which are summarised below.

Integrated care: 69 separate comments and 30 separate comments about the planning for integration between primary and specialist diabetes services, which are summarised in Box 1.

General comments: (37 comments). Participants were invited to give their general comments which were deemed to be important to them in relation to the survey. We were encouraged by the comments that "The survey has worked well" and provide a summary of these comments in Boxes 1 and 2 .

Annual review process and potential barriers to one-stop service: (29 separate comments). These are summarised in Box 3.

The survey comments provided a useful insight into the various levels of integration among primary and specialist diabetes services. A thematic summary of these comments is provided in Boxes 1-3.
Box 2 General comments about integrated diabetes services

1. Integration (General comments)

- A shared vision and the key focus on the importance of the management of a high-risk population to prevent avoidable harm.

- Poor leadership of the diabetes service has unfortunately led to an inability to move forward.

- Fully integrated services would be useful from a patient perspective. It is essential to evaluate the barriers preventing this.

- Integration is definitely the direction of travel. It is often difficult to work across boundaries but diabetes clinicians should take the lead in this alongside 'GP champions'.

- Specialist and primary care working in silos is simply not possible in current times and such working would be of extremely poor quality and costly with associated poor outcomes for people with diabetes.

- Their providers are not willing to let any integration of such systems happen likely due to commercial pressures.

- A national approach to this issue is needed to standardise the IT system

2. IT Integration (General Comments)

- Let's embed a dedicated IT specialist within each diabetes team to start.

- Don't wait for IT to find a solution to better integration. The way forward is to move away from the old model of hospital-based diabetes care and work more closely with primary care in the community.

- Data sharing across primary and secondary care is needed. There are still some barriers in both primary and secondary care.

- Integrated IT support does take central place in establishing an integrated service. Independent trusts and GP services are using a variety of IT support services which do not talk to each other.

- We therefore need IT systems that link primary and secondary care.

- The Scottish Diabetes Group could give an overview of how this functions throughout the country.

\section{Discussion}

Opportunities to reshape clinical services are not common events and usually arise out of the necessity to do things differently, with the aim to improve quality and efficiency of care. While quality improvement initiatives are mostly driven by scientific, societal, economic and technological developments, opportunities sometimes arise at times during adversity, such as the COVID-19 pandemic.

To the best of our knowledge, this is the first survey of its kind in the UK, giving us a unique snapshot of the current state of integration of clinical services for diabetes. The survey represents a sizeable response from the specialist community and we feel that, in general, the data are representative of the UK. We acknowledge that there are several examples of attempts at collaborative working in various areas, ${ }^{13-18}$ although the general uptake in the UK has been relatively slow. This survey provides some insights into the current state and the barriers to such developments.

\section{Opinion of respondents on the degree of clinical service integration in their area}

The main results show that more than $50 \%$ of respondents reported a lack of integration within primary care. While $46 \%$ reported some form of integration of services, only $20 \%$ of those 


\section{Box 3 Annual review process and barriers}

1. Logistics

- GPs should be responsible for providing annual complication screening, whether at their surgery, at retinal screening or another clinical setting, to ensure maximum inclusion

- However, annual review should be done by whoever is seeing the patient at the time and the data should then be accessible for all electronically.

- If secondary care were to take on the annual review for patients under their care, this would not be met.

- Patients love one-stop shops; if you are serious about reaching those who are of working age or are hard to engage with, you have to make the most of one contact as that is the only opportunity you might get in a year.

- The logistics of a one-stop shop may be a bit too much for patients, with concerns that if they miss the one stop they will miss all screening.

- If retinal screening is done more than every 12 months, there should be a one-stop shop without the need for retinal screening.

- The one-stop shop should include all key care processes alongside a pharmacist to aid compliance/concordance of medication (polypharmacy) plus a dietetic review.

2. Barriers and concerns

These fall into GP factors, IT issues and service user factors:

- Clinical continuity. We need to look at how this links in with the rest of diabetes care. So, having someone do an annual review should, ideally, fit in with the team making ongoing adjustments. This may create silos and stop things happening

- Local logistics: GP surgeries perceive a risk of reduced income and GPS wanting to retain control of the annual review process.

- GPs and PNs may resist this element of care being removed from their workstreams. Separating the care processes from those with primary responsibility for care of the individual could lead to a breakdown in the pathway.

- Coordination and dismantling of current set-up; getting all stakeholders on board; administration/management support.

- Lack of IT system integration and infrastructure.

- Setting up IT systems: up-skilling staff to undertake these reviews.

3. Other issues and suggestions

- A one-stop service could be considered a backward step in an era where we should be supporting improved patient engagement through care and support planning.

- Perhaps a 'two-stop shop' should be considered - that is, leaving retinal screening alone but using the Diabetes Eye Screening Programme (DESP) registers to schedule separate urine/blood/foot screens on a different date.

- Post COVID, perhaps we should actually be looking to develop community screening services where all biomedical parameters can be collected/ assessed close to home in an accessible location. "My Diabetes My Way" to allow time to digest the information and inform the formal consultation which should happen 2-3 weeks afterwards.

reported full integration which included most elements of the components outlined earlier.

The survey reported that $72 \%$ of respondents thought the pandemic had slowed down planning for integration and only $18 \%$ thought it had speeded things up. We are unclear of the reasons behind this and can only surmise that overriding priorities during COVID at the front line may have impeded these developments.

Specialist colleagues in diabetes almost universally acknowledge that clinical integration and joined-up working would improve clinical care and is considered optimal for the development of efficient services for people with diabetes in a given locality. However, many expressed dissatisfaction with the lack of wider system support such as lack of clinical leadership, lack of priority by senior management and inadequate funding.

While $A B C D$ would like a deeper understanding of the reasons behind the abovementioned views, we believe that the key missing elements in each locality to improve services and deliver integrated diabetes care may be important.

The findings of this survey indicate that, whilst some CCGs/STPS have taken responsibility to coordinate diabetes services for a locality, this appears to be an exception rather than a universal phenomenon. There remains a feeling among clinicians that commissioners of diabetes services could contribute much more to support the development of integrated clinical services. Examples of good clinical practice delivering integrated care have been previously published. ${ }^{13-19}$

However, the survey data also showed that there are several examples of high levels of integration in some units where local clinical leaders have driven the integration, realising the importance of this to the delivery of high quality and efficient services. The results of this survey show that a very high proportion of specialists are cognisant of the importance of integration but feel constrained in their ability to catalyse successful change. Several barriers were identified which are outlined in the data provided.

This is potentially the first national survey of diabetes integration and there may be a case to repeat this in the future, with more clear benchmarking of the key areas to observe how integration evolves in the future and how it influences outcomes and components of diabetes care such as skill development in primary care. We have previously shown that joined-up working and supporting primary care can lead to upskilling of practices and clinical staff in the provision of levels of care. ${ }^{19}$

Our interpretation is that, while there has been progress since the report on integrated care by the Societies in 2013, a high proportion of primary and specialist diabetes services still continue to work in their silos with little evidence of integrated and joined-up working.

\section{Opinion of respondents on integration of information technology (IT) systems}

The second part of our survey focused on the level of IT integration among hospital systems and also between primary and specialist diabetes teams. The importance of integrated IT systems is recognised as it may allow seamless sharing of clinical information across systems and facilitate improved timely communication between caregivers. Importantly, it has the potential to increase the individual's involvement in their own healthcare. Such systems may avoid duplication and hence expenditure.

While $72 \%$ reported that their hospital had an EPR, a specialist diabetes database was only present in $46 \%$. However, there was evidence of integration among hospital-based IT systems. IT integration between specialist and primary care systems was only reported in a minority of services (29\% 'read \& write' facility, $29 \%$ 
'read only' facility). A substantial proportion reported lack of IT integration among GP/hospital/podiatrists/retinal screening services and reported no shared electronic data between various service providers. IT solutions need to be found to help share data across clinical service units in a consistent manner to improve the present situation observed in this survey.

While nearly half of the specialist teams who replied reported the use of an IT system to deliver specialist diabetes services, low levels of interaction between diabetes systems and the hospital EPR suggest that vital clinical information pertaining to diabetes is not widely visible to other clinicians, posing a substantial clinical risk.

Although $A B C D$ accepts that IT integration is vital to achieve clinical integration as it helps facilitate sharing of clinical information, in isolation it is not enough to achieve the necessary changes. IT systems should be viewed as a vital enabler. Integration is more than the development, acquisition and installation of robust IT systems (in itself a challenge); it requires a joined-up approach with various stakeholders, including those who can enable these changes. This has been highlighted in a recently published government White Paper. The recent formation of primary care networks may facilitate leadership required from primary care. Indeed, the diabetes primary care community has recently launched a document highlighting a renewed vision of joined-up care for people with diabetes. ${ }^{20}$

We observed that, in localities where IT integration has happened, it was based around the use of SystmOne which allows sharing of clinical data. This allows a number of functions which have become so useful in the delivery of diabetes care including rapid communication, instant clinical messaging across teams and e-consultation between primary and specialist teams.

Perhaps the UK diabetes community should help endorse a finite number of excellent IT platforms whereby individual choice can be based on local needs and interconnectivity with existing systems. This may help reduce inertia precluding commitment to IT systems.

\section{Opinion of respondents on annual diabetes clinical reviews}

The final part of our survey focused on the annual review process for diabetes, a process that has been shown to improve outcomes and which has been key to the collection of vital datasets which form a key area for the care planning process. During the COVID19 pandemic there have been huge challenges to the collection of routine data due to the need for shielding of those at highest risk. We were not surprised that up to $60 \%$ of colleagues felt that the arrangements of the annual review process were unsatisfactory and that changes should be made to ensure the process was more efficient, better coordinated and provisions made for feedback to the patients.

Whilst many colleagues were in support of a one-stop service for the annual review process at the retinal screening, it was acknowledged that this raised logistical challenges and also barriers with regard to workforce and flows of finance. The current lack of joined-up IT also proves to be a hurdle.

There was some support for the view that annual reviews should be organised by teams who are primarily responsible for de- livery of diabetes clinical care. In other words, patients who attend specialist diabetes teams routinely should receive annual review processes from these teams. However, significant numbers reported that current systems for annual review as part of the QOF set-up should continue..$^{20}$

To deliver a one-stop service, the type of location was not deemed important as long as it was near to a patient's home and accessible with some flexibility. Coordination and sharing of the data collected during annual review was deemed to be important. Therefore, the location of one-stop services could be determined by service users and community healthcare teams.

Integrating care has meant that more people are seeing the benefits of joined-up care between GPs, care at home and in care homes, community health services, acute trusts and mental health services. For staff, it has enabled people to work outside individual organisational silos, deliver more user-centred and personalised approaches to care, and identify and help tackle barriers preventing optimal care for people with diabetes. It enables greater ambition on tackling health inequalities and the wider determinants of health.

The results of this survey point out clearly that we are some way off the universal existence of an acceptable standard of integrated diabetes services in the UK. Solutions need to be developed and put in place to address this urgently; fortunately, none of the barriers are insurmountable.

$A B C D$ believes that the experience of the pandemic has made the case for integrated care even more strongly and believes the insights from this survey should serve as a stimulus for wider discussions among stakeholders. The results of this survey should form the basis for making firm recommendations to commissioners for improving the state of clinical and IT integration in the UK, similar to that in Scotland.

We believe urgent prioritisation and resources are needed from NHS England to develop truly integrated diabetes services. We hope the planned legislation based on the government White Paper will facilitate delivery of this and call upon our membership to help drive this process.

Government plans for integrated services have been outlined recently in a White Paper for integrated services, highlighting the two principal forms of integration which will need to be underpinned by the legislation:21

(a) Integration within the NHS to remove some of the cumbersome and unnecessary boundaries which inhibit collaboration and to make working together a high-level organising principle.

(b) Greater collaboration between the NHS and local government, as well as wider delivery partners, to deliver improved outcomes to health and wellbeing for local people.

In theory, this should enable different parts of the health and care system to work together effectively in a way that will improve outcomes and address inequalities. Clearly, the details of this crucial legislation have the mechanism to facilitate true integration.

The NHS has experienced several cycles of high-level organisational changes and some of these have led to greater bureaucracy and added barriers to joined-up and collaborative working, which 
remains the essence of integrated care. Keeping this in mind, the White Paper plans to give additional power to the Secretary of State for Health and Social Care to intervene in how NHS England operates.

The White Paper proposes substantial legislative changes which aim to:

- Make permanent the innovations that COVID-19 has accelerated and encourage the system to improvise new and better ways of working.

- Integrate healthcare in England by enshrining integrated care systems in law.

- Reduce bureaucracy and create flexibility.

- Improve NHS England accountability and enhance public confidence.

We welcome the recent signals from government with regard to their determination to ensure that public health, social care and healthcare work more closely together in the future than ever before. We recommend simplicity, clarity and commitment for legislative changes to help delivery of these objectives to be achieved - namely, to deliver true integrated care. We hope the planned legislation based on the government White Paper will ultimately facilitate delivery of this promise and we call upon our membership to step into the driving seat.

\section{Recommendations}

Based on the results of this important survey, $A B C D$ recommends that, in addition to the enablers for integrated care outlined, clinical services in a given locality should aspire to at minimum:

1. A joined-up approach to planning and delivery of diabetes services among commissioners, specialist diabetes teams and primary care.

2. The aim should be to improve quality of diabetes services to a higher level and to improve clinical outcome of individuals with diabetes.

3. A designated lead who will be responsible for overseeing that integrated services are developed and allowed to expand in a given locality (ie, there is governance and accountability for this).

4. Each diabetes specialist team should be supported by a dedicated IT system/s and diabetes database in their units.

5. Specialist diabetes IT systems must interact and be integrated with local EPR and primary care systems, allowing easy sharing of data for ease of delivery of clinical care.

6. There should be a high-level NHS mandate for the above recommendation during future re-organisation of chronic disease management in the UK, as suggested in the recent White Paper.

\section{Limitations of the survey}

1. No service user involvement during survey design. Hopefully, discussion fuelled by this paper will allow a more structured dialogue with service users in future surveys. Any future recommendations for redesign of services must involve patients and careers.

2. The survey did not ask if the IT system allowed direct patient access to their data (in either 'read-only' or 'read and write' format) or issues related to data protection.

\section{Key messages}

- This survey shows, that the level of integration among primary and specialist diabetes teams is far from ideal and leaves much room for improvement

- Many Primary and Specialist Diabetes services continue to work in their own silos and in isolation, which is not delivering good quality diabetes care our service users deserve

- Many Specialist diabetes services do not have a dedicated diabetes specific IT system, which is essential to the delivery of diabetes care in this era

- Hospital based EPR (Electronic Patient Records) systems and the diabetes system do not communicate with each other, therefore limiting the flow of clinical information from one to the other system, creating a degree of clinical risk

- The findings of this survey, should provide a vital platform for discussions among wider diabetes teams and commissioners as to how integrated care can be developed in local health economies.

3. The survey was limited to the $A B C D$ membership, which may skew the responses.

4. The information provided is based on the view of individual clinicians rather than data collected using a more structured and robust method, and is open to over- or under-reporting of the true picture.

5. The overall survey response was low, which may limit the conclusions of the survey.

6. There were some leading questions which might have biased the reply from the respondent.

7. As the participation was voluntary, we cannot exclude a self-selection bias.

8. Under-representation of some areas (eg, East Midlands, North East, Northern Ireland).

\section{Strengths of the survey}

1. The survey questions were designed after significant discussion within the executive committee, a group of experienced diabetologists. We asked contemporary and targeted questions; answers to these are likely to help future diabetes care.

2. This is the first comprehensive attempt to acquire a view from all diabetes hospital services in the country. A representation of 73 different diabetes units with different levels of integration provides a picture which is likely to be reliable.

3. The qualitative aspect of this survey enriches the practical value and applicability of the survey.

4. Those constructing future surveys in this area can learn from omissions and the limitations and strengths of the present survey. 
5. The survey was collected during the pandemic and revealed serious gaps in our existing services, especially integration with primary care and IT solutions. The survey captured the current and new beliefs informed by the challenges of diabetes care during the pandemic.

6. The survey represents views of specialists across four nations, which will allow us to learn from the strengths/weaknesses of these services.

Acknowledgements We are very grateful to Dr Rustam Rea (Oxford Diabetes Unit) for valuable and astute comments regarding this survey and important suggestions during preparation of this manuscript.

\section{Conflict of interest None. \\ Funding None.}

\section{References}

1. Choudhary P, Wilmot EG, Owen $K$, et al. A roadmap to recovery: $A B C D$ and Diabetes UK position statement on adult diabetes services in the postCOVID-19 era. Diabet Med 2021;38:e14462. http://dx.doi.org/10.1111/ dme.14462

2. Nagi DK, Wilmot EG, Owen K, et al. ABCD position statement on risk stratification of adult patients with diabetes during Covid-19 pandemic. $\mathrm{Br} J$ Diabetes 2021;21(1). http://doi.org/10.15277/bjd.2021.282

3. Nagi D, Choudhary P, Wilmot EG, Winocour P. Supporting people with diabetes during the COVID-19 pandemic without face-to-face appointments. Br J Diabetes 2020;20(1):1-4. https://doi.org/10.15277/bjd.2020.246

4. Mills L, James J, Hicks D, et al. A collaborative overview and insight into diabetes services before and after the COVID-19 pandemic. https://trenddiabetes.online/wp-content/uploads/2020/09/Covid_Report_TREND_v43.pdf (accessed August 2021).

5. $A B C D /$ Diabetes UK. Maintaining acute diabetes services in response to COVID-19. Prepared by the National Diabetes Inpatient COVID Response Team (Rayman G (Chair), Lumb A, Kennon B, et al). https://abcd.care/sites/ abcd.care/files/site_uploads/Maintaining\%20Inpatient\%20Teams_FINAL_7. 4.pdf (accessed 12 June 2021).

6. Goenka N, Turner B, Vora J, Diabetes UK Task and Finish group. Commissioning specialist diabetes services for adults with diabetes: summary of a Diabetes UK Task and Finish group report. Diabet Med 2011;28(12):1494500. https://doi.org/10.1111/j.1464-5491.2011.03410.x

7. The King's Fund. Integrated care: our position. https://www. kingsfund.org.uk/projects/positions/integrated-care (accessed 16 July 2021).

8. The King's Fund. The next steps towards integrated care. https://www.kingsfund.org.uk/publications/next-steps-towards-integrated- care (accessed 16 July 2021).

9. Best practice for commissioning diabetes services: an integrated framework: https://diabetes-resources-production.s3-eu-west-1.amazonaws. com/diabetes-storage/migration/pdf/best-practice-commissioning-diabetesservices-integrated-care-framework-0313.pdf (accessed 12 June 2021)

10. Tan GD, Kozlowska O, Rea RD. Delivery and organisation of diabetes care: integrated care. Medicine 2018;47(2):127-30. https://doi.org/10.1016/ j.mpmed.2018.11.008

11. GE Health Care Consulting. Insights: 10 key indicators of clinical integration success. https://www.gehealthcareconsulting.com/insights/10-key-indicators-of-clinical-integration-success (accessed 12 June 2021).

12. Nagi D, Wilson J. Integrated diabetes care: The Wakefield Diabetes Service redesign. Practical Diabetes 2011;28(7):310-11.

13. Nagi D, Wilson J, Kadis T, Jenkins R. Diabetes service redesign in Wakefield HMS high-security prison. Diabetes Prim Care 2012;14(6):344-50.

14. Cranston I, Meeking D, Kar P, Nicholson EJ, Chhapia R, Cummings M. The Super Six Diabetes Model: creating a legacy? Diabetes Prim Care 2018; 20(1):19-22.

15. Kozlowska O, Attwood S, Lumb A, Tan GD, Rea R. Population health management in diabetes care: combining clinical audit, risk stratification, and multidisciplinary virtual clinics in a community setting to improve diabetes care in a geographically defined population. An integrated diabetes care pilot in the North East locality, Oxfordshire, UK. Int J Integrated Care 2020; 20(4):21. https://doi.org/10.5334/ijic.5177

16. Sachar A. How important is mental health involvement in integrated diabetes care? The Inner North West London experience. London J Primary Care 2013:5(1):63-7.

17. McHugh S, O'Mullane M, Perry IJ, Bradley C. Barriers to, and facilitators in, introducing integrated diabetes care in Ireland: a qualitative study of views in general practice. BMJ Open 2013;3(8):e003217. https://doi.org/ 10.1136/bmjopen-2013-003217

18. Nagi D. An introduction to diabetes service redesign in Wakefield: https://www.guidelinesinpractice.co.uk/diabetes/an-innovative-diabetesservice-redesign-in-wakefield/352883.article (accessed 2 Aug 2021).

19. GOV.UK. Integration and innovation. Working together to improve health and social care for all. February 2021. https://www.gov.uk/government/ publications/working-together-to-improve-health-and-social-care-for-all (accessed 2 June 2021).

20. BMA/NHS. 2020/21 General Medical Services (GMS) contract Quality and Outcomes Framework (QOF): guidance for GMS contract 2020/21 in England. https://www.england.nhs.uk/wp-content/uploads/2020/09/C0713202021-General-Medical-Services-GMS-contract-Quality-and-OutcomesFramework-QOF-Guidance.pdf (accessed 16 July 2021).

21. Best practice in the delivery of diabetes care in the primary care network. April 2021. https://diabetes-resources-production.s3.eu-west-1.amazonaws.com/resources-s3/public/2021-04/Diabetes-in-the-Primary-CareNetwork-Structure-April-2021_with-logos.pdf (accessed 16 July 2021). 